\title{
Recursos tecnológicos aplicados al desarrollo del tiempo verbal pasado en inglés
}

\author{
Technological resources applied to the development of the Past Tense in \\ English \\ Rubén O. Quintero-Espinoza ${ }^{a}$, Jesús E. Fong-Flores ${ }^{b}$, Laura E. Fierro-López $^{c}$, Tatiana E. \\ Galván-De la Fuente
}

\begin{abstract}
:
Linguistic awareness of the Past Tense in English is indeed a complex issue for the learner. It is understood that in the process and access to English as a target language, there are diverse representations of errors that are considered transitory in nature. This research proposes the use of technological resources applied to the learning and to the development of the Past Tense as it is evidenced by the analysis, classification, and description of the written errors that learners from the Core Curriculum at the Language Faculty of Mexicali of the Autonomous University of Baja California. Furthermore, the proposal is also grounded on the results of a survey that revealed the learners' perception towards the use and preference of technological resources. This is a mixed method, descriptive and non-experimental research.
\end{abstract}

\section{Keywords:}

Error analysis, error typology, technological resources, verbal tense

\section{Resumen:}

La conciencia lingüística desde la comprensión del tiempo verbal pasado, representa un punto de complejidad para el estudiante. Se entiende que, en el proceso y acceso al inglés como lengua meta existen representaciones del error que son elementos de orden transitorio. Nuestra investigación propone recursos tecnológicos aplicados a la comprensión y desarrollo del tiempo verbal pasado, que se sustentan a partir del análisis, clasificación y descripción de errores escritos por estudiantes del tronco común de la Facultad de Idiomas, Mexicali de la Universidad Autónoma de Baja California. Además, la propuesta se fundamenta a partir de la encuesta de opinión que muestra la percepción del estudiante hacia el uso y preferencia de los recursos tecnológicos. Se realizó una investigación de enfoque mixto, de alcance descriptivo y de diseño no experimental

\section{Palabras Clave:}

Análisis de errores, tipología del error, recursos tecnológicos, tiempo verbal

\section{INTRODUCCIÓN}

La presente investigación propone recursos tecnológicos aplicados a la comprensión y desarrollo del tiempo verbal pasado en inglés. Para este objetivo se realizó el análisis, clasificación y descripción de errores escritos que presentan los estudiantes de la asignatura Análisis y Disertación de
Textos en la Segunda Lengua del tronco común de la Facultad de Idiomas, Mexicali de la Universidad Autónoma de Baja California.

Se reconoce el estado actual del estudiante quien, previo al ingreso a una etapa disciplinar, debe consolidar ciertas bases lingüísticas fundamentales en su formación profesional. Por

\footnotetext{
${ }^{a}$ Corresponding author, Universidad Autónoma de Baja California, Email: octavio.quintero@uabc.edu.mx

${ }^{b}$ Universidad Autónoma de Baja California, Email: jfong@uabc.edu.mx

${ }^{b}$ Universidad Autónoma de Baja California, Email: octavio.quintero@uabc.edu.mx

${ }^{b}$ Universidad Autónoma de Baja California, Email: laurafierro@uabc.edu.mx
} 
ello dentro de la composición de este trabajo, se identificaron errores que determinan y justifican la construcción de nuestro espacio tecnológico.

Por lo que refiere a la tipología del error considerada para este estudio, misma que se sustenta en los párrafos posteriores, tiene como punto de partida el error intralingual, esto debido a los intereses propios de esta investigación. La representación del error intralingual se deriva de una falta de concientización lingüística y no, propiamente, de la presencia de otras lenguas. De esta forma la conciencia lingüística, desde la comprensión del tiempo verbal pasado en inglés, representa un punto de complejidad inicial para el estudiante en formación. Siendo necesario reflexionar que dentro del proceso y acceso al inglés como lengua meta existen representaciones de un error transitorio.

Es así que el estudio parte de las siguientes interrogantes: ¿los estudiantes presentan construcciones lógicas hacia la utilización del tiempo verbal pasado en la producción escrita de textos narrativos?, ¿qué estrategias o recursos tecnológicos debe implementar el profesorado para el desarrollo, comprensión y uso del tiempo verbal?

La identificación del error y su tipología ofrecen las herramientas base para configurar ambientes de aprendizaje mediados por recursos tecnológicos. No obstante, independientemente de la identificación de necesidades lingüísticas, creemos que cualquier propuesta educativa parte de la percepción y opinión del estudiante en formación. Este estudio comprende un enfoque mixto, en primera instancia mediante una prueba diagnóstica (que conforma el corpus de los textos escritos de los estudiantes) y la encuesta de opinión para analizar la percepción de los estudiantes en relación al uso y preferencia de recursos tecnológicos. Siendo una investigación de alcance descriptivo y basada en un diseño no experimental.

\section{REVISIÓN DE LA LITERATURA.}

La lengua meta y su aprendizaje, sostiene puntos de complejidad transitoria hacia un nuevo sistema lingüístico para el individuo. Cuando hablamos de gramática y su proceso de enseñanza, debemos poner sobre la mesa el fomentar una competencia gramatical. Basado en Fasold y Connor $(2014$, p.9) se define "the part of the system that allows speakers to produce and interpret grammatical sentences is called grammatical competence".

Para el profesorado de lengua, independiente de las adecuaciones metodológicas o enfoques para la enseñanza de lenguas, abordar la enseñanza de los tiempos verbales puede representar un reto. Donde habría que considerar distintas figuras de la gramática no como un conocimiento aislado, por lo contrario, referir a dos puntos medulares: conocimiento gramatical y habilidad gramatical (Richards y Reppen, 2014).

Lo descrito pone a consideración que en el aprendizaje del compuesto gramatical, existen distintos factores que intervendrán: el contexto, el proceso de enseñanza y aprendizaje, condiciones en que se da el aprendizaje, entre otros (Stern, 1983).

Por ello, el interés debe enfocarse en desarrollar competencias y habilidades que permitan al individuo (estudiante) dar un uso correcto de la lengua meta; es ahí que se puede hablar de una competencia comunicativa. Sañudo (2013) la define como convicciones lingüísticas que parten de la coherencia y/o cohesión, momentos discursivos del hablante, y de una apropiación de lo formal e informal según los distintos espacios y contextos. En el cual, valdría complementar que la lengua tiene características únicas asociadas con su forma y función.

Es en este momento que se debe comprender cuáles son las aproximaciones didácticas y pedagógicas, por parte del profesorado, hacia la construcción de una competencia integral en la lengua meta. La apropiación de una competencia de la lengua se sostiene a razón de cuatro subcompetencias interrelacionadas: competencia lingüística (gramatical), sociolingüística, discursiva, y estratégica (Canale, 1983). Los puntos descritos muestran un acercamiento a la lengua como un elemento global, no obstante, resulta relevante abordar a la lengua a razón del contexto de enseñanza y al proceso aprendizaje.

Saborio (2019) expresa que hacia el desarrollo de una competencia gramatical debe plantearse un acercamiento orientador y constructivo. Para ello, cobra relevancia el hecho de que el profesorado realice adaptaciones a enfoques o metodologías aplicadas desde un marco contextualizado. De esta forma, el proceso conlleva a que el estudiante sea consciente de su aprendizaje y desarrolle distintas capacidades entorno a la lengua meta.

Tras este sucinto acercamiento al proceso gramatical, se toma la noción de la concientización lingüística. Lyster (2001) expone que el término de conciencia aborda los distintos niveles lingüísticos: ortográfico, fonológico, morfológico, sintáctico y/o semántico. Comprendemos que el término se refleja en los distintos niveles de la lengua y es dependiente de su interpretación. En un acercamiento más específico, Bolitho, Carter, Hugh, Ivanic, Masuhara y Tomlinsones (2003) expresan: "language awareness is a mental attribute which develops through paying motivated attention to language in use, and which enables language learners to gradually gain insights into how languages work" (p. 251).

Esta postura constituye que la gramática posee un rol medular en el proceso de aprendizaje de la lengua meta. La conciencia lingüística alienta al estudiante a hacer uso de la gramática en la vida cotidiana, así como al hecho, de construir un interés 
por aprender y ser responsable del aprendizaje. Sin duda, reconocemos que hablar de conciencia lingüística y del aprendizaje es aún punto pendiente dentro del aula.

La conciencia lingüística parte que el individuo, nativo hablante del español, reconozca a la lengua meta (inglés) desde un hecho consciente y analítico; de reconocer al sistema lingüístico de forma independiente y a razón de las reglas que lo rigen. Ciertamente, las distintas propuestas al concepto de bilingüismo (Bloomfield, 1933; Cummins, 1976; Haugen, 1953; Hakuta y Díaz, 1985) o multilingüismo (Andrews, 2014; Edwards, 2007; Flynn, 2016) muestran que el bilingüe o multilingüe surge de esta conciencia; de normas y estructuras definidas que comprende una lengua meta. Aunado a lo descrito, se debe acotar esta noción al término de la metalingüística. Dentro de esta acepción, Bialystok (1988) proponía la existencia de dos aspectos importantes a considerar: el análisis del conocimiento lingüístico y control de procesamiento lingüístico. Para dejar este aspecto claro, la autora indica (p.561):

Analysis of linguistic knowledge is the skill component responsible for the structuring and explication of linguistic knowledge.

Control of linguistic processing is the executive component responsible for directing attention to the selection and integration of information. In information-processing terms, the control component corresponds to executive processes or metacomponents (fluid ability).

Todo lo mencionado hasta ahora, pone la mirada hacia la conciencia metalingüística la cual brinda un punto de valor a este trabajo. Roehr (2007) reconoce tres puntos interesantes que valdría expresar: una conciencia no refiere a símbolos del lenguaje sino a un conocimiento amplio, que las palabras tienen un significado propio desde distintas dimensiones y, que el lenguaje está basado en reglas y estructuras dependientes de su uso.

Queda claro que en la identificación de una conciencia lingüística o metalingüística, la función principal parte del cómo se promueve en el aula de LE. El objetivo desde luego es proveer una visión más holística y por consiguiente, comprender que las estructuras gramaticales son y serán recursos lingüísticos que proveen una comunicación asertiva.

\section{El análisis de errores}

Hacia 1967, Corder realizó los primeros estudios sistemáticos de errores de estudiantes de una L2, dado que hasta esa fecha las únicas investigaciones realizadas eran aquellas donde se contrastaban sistemas lingüísticos de la L1 con L2. Con la finalidad de descubrir diferencias y similitudes entre ambas lenguas para posteriormente predecir los posibles errores del estudiante a lo largo su aprendizaje. Lo anterior bajo la premisa que en lo que respecta al error, no todos los errores deben reconocerse como fallos en la producción de la lengua (Corder, 1967).

A diferencia del análisis contrastivo, el Análisis de Errores (AE) no consistía en comparar la lengua materna y lengua meta, sino en producciones orales y/o escritas que darían un mejor entendimiento al uso de la lengua de forma independiente. La contribución de Corder muestra un punto conveniente al establecer una serie de pasos para el análisis del error: identificación de los errores en su contexto, clasificación y descripción de los errores, explicación de su origen, evaluación del error y su posible tratamiento. Este último punto, hace alusión a cómo atender al error mediante recursos tecnológicos aplicados al desarrollo de la lengua.

En seguimiento a la propuesta de AE, se observó que los errores reflejaban estrategias universales de aprendizaje. De ahí nace la aportación más importante de esta corriente: el cambio de visión del error. Los errores empezaron a ser considerados como un factor provechoso en el aprendizaje porque constituían un paso ineludible en el camino de apropiación de la nueva lengua (Martín et al., 2005, párr.4). Sin embargo, es importante establecer una definición conveniente pues existen diferentes fuentes que ahondan al respecto.

La noción del AE, expresa su funcionalidad para revelar los errores cometidos por los estudiantes, tanto en la escritura como en el habla (Richards, 1975). Roldán (1979) define al análisis de errores en la enseñanza y evaluación como una forma para medir el progreso, registrando y clasificando los errores cometidos por un alumno o por un grupo de alumnos. A este postulado se requiere agregar que el profesor juega un papel importante en la detección de errores, puesto que de la revisión se presenta un panorama más claro sobre la producción de la lengua; mismo que deriva en una realimentación o posible tratamiento.

Rustipa (2011) propone que un hallazgo clave del análisis de errores, ha sido que muchos errores de los alumnos son producidos por inferencias defectuosas sobre las reglas (generalizaciones de las normas y reglas de la lengua). Aunque el AE se fundamenta a partir de un histórico que se apropió de ciertas posturas, la búsqueda de una teoría general en los errores se ha ampliado hacia nuevas perspectivas. En sintonía, se puede decir que el error es un producto particular del estudiante y que deberá analizarse dentro del desarrollo de la lengua; oral o escrita.

Akbar (2012) expresa que el AE es un análisis lingüístico que se centra en identificar los errores que cometen los estudiantes. Consiste en una descripción particular que enfatiza la importancia de los errores de alumnos en el segundo idioma. Es decir, de insistir que el error adquiere un valor dentro del proceso de enseñanza y aprendizaje. 
Para cerrar este apartado, dejar claro que el $\mathrm{AE}$ se ha redefinido y adaptado a los intereses propios del profesorado; donde toma un nuevo acercamiento a la lengua y al estudiante. En un primer punto, de buscar un mejor entendimiento del error en función su teorización y/o clasificación, y por otra hacia el tratamiento que podría brindarse a partir de distintos recursos educativos; en este caso mediado por tecnológicas aplicadas a la educación.

\section{El error}

El análisis del error constituye una base teórica pero, a su vez, es de índole metodológica. Desde 1975 Richards sostenía la presencia de dos categorías medulares hacia la noción del error: errores interlinguales e intralinguales (éste último considerado en esta investigación). Para ampliar esta cuestión, el autor mencionaba que los errores interlinguales surgen a razón de la interferencia lingüística de otras lenguas, véase como materna $\mathrm{u}$ otra lengua con la que el individuo esté en contacto. Por lo que refiere a la tipología intralingual se entiende como errores transitorios y a razón de un aprendizaje en proceso.

Independiente de la categorización, se tienen contribuciones con la postura del error de Dulay, Burt, y Krashen (1982) que entienden como error a una desviación sistemática de las normas de la lengua. Este expuesto plantea la interrogante, ¿hasta qué punto es posible incidir en desviaciones de la norma en una lengua?

El error expresa una línea distante o aproximada al aprendizaje esperado como evidencia de la producción del individuo en lengua meta; a partir de sus propias reglas. Desde la categorización del error, conviene añadir otras figuras que separan la tipología interlingual e intralingual. James (2013) hace un razonamiento más preciso tras mostrar: a) el error interlingual se presenta por la influencia de las lenguas que conoce el individuo, b) e intralingual hacia generalizaciones que presenta el estudiante en ausencia de una conciencia lingüística.

Richards y Schmidt (2010) muestran "the use of a linguistic item in a way which a fluent or native speaker of the language regards as showing faulty or incomplete learning" (p.201). Esta aportación hace una redefinición del posicionamiento del error, también evidente en otros autores (Brown, 2000; Richards, 1974). Desde el panorama actual del AE, visto como proceso sistematizado, sería apropiado reconocer estas variaciones del error adaptadas al propósito del investigador.

Esta intención de reconocer al error desde una tipología específica, parte del hecho que los errores requieren de una descripción que permita reconocer las competencias del estudiante en una LE. Ciertamente el error desde un criterio etiológico, es decir desde su descripción, permite que el docente tenga un mejor entendimiento y valoración del error.

\section{Aprendizaje asistido por tecnologías}

Nuestro interés, es reconocer a las tecnologías como un recurso que se extiende a las distintas áreas de conocimiento y desde intereses particulares. Su introducción dentro de los contextos formales de aprendizaje se orienta como: proveedor de nuevos conocimientos (Adell, 1997), herramientas de apoyo didáctico (Navarro, 2011), aplicación del aprendizaje en contextos reales y simulados (González y Ortíz, 2011).

El Aprendizaje de Lenguas Asistido por computadora (CALL por sus siglas en inglés) representa una herramienta, no necesariamente de orden metodológica, que exhorta a comprender las intenciones del recurso y cómo se dará uso de él. Debe quedar claro que la utilización del recurso tecnológico no remplaza al docente, lo opuesto, es un medio mediante el cual el profesorado y el estudiante darán un uso como facilitador de acceso al aprendizaje.

La contribución consecutiva surge en Beatty (2003), define al aprendizaje asistido como el proceso y uso de recursos tecnológicos en el aprendizaje que inciden en la mejora y desarrollo de la lengua. Esta aproximación muestra una línea similar a la descrita por distintos autores (Chapelle, 2001; Levy, 1997), tras reconocer al recurso asistido como mediador en el aprendizaje.

Para el campo de las lenguas, se muestra al aprendizaje asistido por computador como la mediación hacia el estudio de la enseñanza y aprendizaje basada en recursos tecnológicos (Levy y Stockwell, 2006). El uso de herramientas tecnológicas permite crear un entorno flexible de aprendizaje, que parte de necesidades específicas del estudiante y alcances esperados en la lengua. En este sentido, se retoma la idea del docente innovador que provee de espacios distintos hacia la adecuación y adaptación de los métodos o enfoques aplicados en el aula. Del mismo modo, Egbert y Petrie (2006) expresa que a través del CALL los estudiantes tienen acceso a un aprendizaje mediante un recurso basado en tecnologías informáticas de acceso diverso.

Una vez identificada la intención del aprendizaje asistido, es necesario detallar lo propuesto por Martín (2012). El autor muestra a continuación los primeros usos de la Enseñanza de Lenguas Asistido por Ordenador (ELAO):

ELAO conductista (Behaviouristic CALL), basada en las teorías pedagógicas de Skinner (1957); ELAO comunicativa (Communicative CALL), en línea con el enfoque comunicativo en enseñanza de idiomas; y ELAO integradora (Integrative CALL), influida por la pedagogía socio-cognitiva, centrada en actividades del tipo task-based, project-based y content-based y marcada por la aparición de materiales multimedia e Internet (Davies, 2007), los 
cuales favorecen la interactividad y el trabajo colaborativo (p.204).

Desde la figura diacrónica y de cambio del concepto, evidenciar que la condición más importante del aprendizaje asistido reside en redefinir su enfoque y aplicación a los distintos contextos formales y no formales de aprendizaje. Tal como expresan Chapelle y Hegelheimer (2004) "The need has never been greater for teachers with basic technological skills who understand the capabilities and limitations of technology in teaching and who accept responsibility for critically examining the options and their implications" (p.313).

Para cerrar este apartado, dejar claro que hacia el desarrollo de ambientes de aprendizaje asistidos por TICs se requiere partir de ciertos pasos: a) un análisis de necesidades, y b) un marco teórico y metodológico que considera a la enseñanza y aprendizaje (Hubbard, 2009).

\section{Metodología}

El objetivo de la investigación es proponer estrategias de enseñanza para el desarrollo del tiempo verbal mediadas por recursos tecnológicos. Esta contribución surge del análisis de las producciones escritas en inglés y el desarrollo del tiempo verbal pasado desde la narrativa. La metodología empleada en el estudio es de alcance descriptivo con diseño no experimental. Aguilera (2013) menciona que la metodología describe una lógica que sustenta los pasos requeridos que se tienen que transitar para llegar al resultado que se persigue. La investigación se realizó con un enfoque mixto para la obtención de datos que permitió el análisis de errores, su tipología (intralingual) e interpretación. Se identificaron las incidencias y frecuencias en las producciones escritas de los estudiantes; así mismo, mediante una encuesta para analizar la percepción de los estudiantes y aproximarse al uso y preferencia de recursos tecnológicos.

\section{Muestra}

El estudio se centró en la identificación del error intralingual en las producciones escritas en inglés realizadas por estudiantes en la asignatura Análisis y Disertación de Textos en la Segunda Lengua durante el semestre 2020-1. Esta materia se ubica en el segundo semestre correspondiente al tronco común de las carreras de licenciatura ofertadas en la unidad académica.

Se aplicaron pruebas diagnósticas a dos grupos con el propósito de realizar una descripción más amplia del proceso de comprensión del tiempo verbal pasado, sin la intención de compararlos. Los participantes, que permanecen en total anonimato, cuentan con un rango de edad 18-25 años, nativohablantes del español; otros se descartan del estudio. Lo anterior hacia el reconocimiento y posicionamiento del inglés como lengua meta. La investigación se adhiere a un muestreo por conveniencia.

\section{Procedimiento}

Como se indica se realizaron pruebas diagnósticas a dos grupos para identificar recurrencias y frecuencias en el error. La prueba diagnóstica representa el punto de partida con el objetivo de conformar el corpus para su posterior análisis. Se les indicó elaborar un texto narrativo con un tema abierto, a su elección, en el tiempo verbal pasado.

Posteriormente, se hace una revisión del corpus de los textos escritos a través de la estructura del análisis de errores desde la tipología del error intralingual. Cabe aclarar que el procedimiento para la identificación del tiempo verbal no fue automatizado, se realizó la lectura de cada producción escrita, se identificaron los errores y se tipificaron según las categorías consideradas. En este entendido la propuesta contempla obtener información de tres variables principales: cantidad, cantidad por categoría y tipos de errores.

\begin{tabular}{|l|l|}
\hline Criterio & Descripción \\
\hline Cantidad de errores & $\begin{array}{l}\text { Se identifica la cantidad total de errores } \\
\text { identificados en las producciones } \\
\text { escritas. }\end{array}$ \\
\hline $\begin{array}{l}\text { Cantidad por } \\
\text { categoría }\end{array}$ & $\begin{array}{l}\text { Se identifican los errores en past simple, } \\
\text { past continuous, past perfect, past perfect } \\
\text { continuous. }\end{array}$ \\
\hline $\begin{array}{l}\text { Tipos de errores } \\
\text { desde el criterio } \\
\text { (etiológico) } \\
\text { intralingual }\end{array}$ & $\begin{array}{l}\text { Hipergeneralización } \\
\text { Ignorancia de las restricciones de las } \\
\text { reglas } \\
\text { Aplicación incompleta de las reglas }\end{array}$ \\
\hline
\end{tabular}

Tabla 1. De criterio y descripción del error

Por lo que refiere a la tipología, la intención reconoce una subcategorización del error intralingual: errores por hipergeneralización, ignorancia de las restricciones de las reglas, y aplicación incompleta de las reglas. Para esclarecer este punto, recurrimos a lo propuesto por Alexopoulou (2006):

\begin{tabular}{|l|l|}
\hline $\begin{array}{l}\text { Subdivisión del } \\
\text { error intralingual }\end{array}$ & Descripción \\
\hline $\begin{array}{l}\text { Los errores por } \\
\text { hipergeneralización }\end{array}$ & $\begin{array}{l}\text { La hipergeneralización es una estrategia } \\
\text { creativa mediante la cual el aprendiente } \\
\text { intenta establecer paralelismos y } \\
\text { analogías. Lo que induce al error es la } \\
\text { falsa generalización de una regla. }\end{array}$ \\
\hline $\begin{array}{l}\text { Ignorancia de las } \\
\text { restricciones de las } \\
\text { reglas }\end{array}$ & $\begin{array}{l}\text { Refleja el fracaso por parte del } \\
\text { aprendiente para observar las } \\
\text { limitaciones en la aplicación de una regla } \\
\text { en contextos en que ésta no se puede } \\
\text { aplicar. }\end{array}$ \\
\hline $\begin{array}{l}\text { Aplicación } \\
\text { incompleta de las } \\
\text { reglas }\end{array}$ & $\begin{array}{l}\text { Se trata de una aplicación parcial de } \\
\text { reglas; refleja el fracaso por parte del } \\
\text { estudiante para desarrollar plenamente } \\
\text { una estructura conocida; puesto que el } \\
\text { aprendiente simplifica el sistema. }\end{array}$ \\
\hline
\end{tabular}

Tabla 2. Tipología del error considerada 
Para la encuesta que determina la percepción y uso de la tecnología, se consideraron los siguientes elementos: a) identificación de puntos de complejidad en el aprendizaje de la lengua, b) interés por la lengua, c) interés por la gramática de la lengua, d) razón por la cual existe o no un interés por la gramática, e) autoevaluación de la gramática (reactivo que comprende distintas subcategorías).

\section{Resultados y discusión}

La prueba diagnóstica, reconoce cuáles son las competencias por parte del estudiante en la construcción de una narrativa; sin parámetros rigurosos. Es decir, se solicitó la elaboración de un texto escrito que tenía como lineamientos una subdivisión a razón de introducción, desarrollo y conclusión, y por otro lado, de presentar una cantidad determinada de palabras.

Una vez reiterado ese aspecto, se muestran los datos obtenidos en la prueba diagnóstica. En primer término, de los dos grupos se obtuvo un total de 47 producciones escritas. El procedimiento para el análisis fue:

a. recopilación de las producciones escritas para la conformación del corpus.

b. identificación de errores por tiempo verbal y desde la tipología considerada en el estudio.

c. descripción de errores.

Los datos recuperados posicionan un estadío en el aprendizaje del estudiante, tras evidenciar que el error se encontraba en las distintas categorías del tiempo verbal. De un total de los 120 errores (incidencias) identificados en los distintos textos existieron representaciones particulares:

\begin{tabular}{|l|l|l|}
\hline Criterio & Frecuencia & $\%$ \\
\hline Cantidad & 120 errores & $100 \%$ \\
\hline Cantidad por categoría & 103 & $86 \%$ \\
\hline Past simple & 1 & $1 \%$ \\
\hline Past continuous & 16 & $13 \%$ \\
\hline Past perfect & 0 & 0 \\
\hline Past perfect continuous & 35 & $29 \%$ \\
\hline Tipos de errores intralinguales & $68 \%$ \\
\hline $\begin{array}{l}\text { Hipergeneralización de las } \\
\text { restricciones de las reglas }\end{array}$ & 82 & $3 \%$ \\
\hline $\begin{array}{l}\text { Aplicación incompleta de } \\
\text { las reglas }\end{array}$ & 3 & \\
\hline
\end{tabular}

Tabla 3 Descripción general de errores

Ciertamente, los errores dentro del análisis no pueden verse únicamente desde un orden generalizado. Por lo contrario, se mostrará a continuación una subclasificación de errores a partir del tiempo verbal: past simple, past continuous, past perfect, past perfect continuous. Debido a la extensión de los distintos ejemplos recuperados por parte del análisis, se mostrarán los más representativos y que expresan una evidencia de la tipología descrita.

\begin{tabular}{|c|c|c|c|c|c|c|}
\hline \multirow{3}{*}{$\begin{array}{l}\text { Cantidad } \\
\text { por } \\
\text { categoría } \\
\text { Past simple }\end{array}$} & \multicolumn{6}{|c|}{ Frecuencias Absolutas } \\
\hline & \multicolumn{2}{|c|}{ Hipergeneralización } & \multicolumn{2}{|c|}{$\begin{array}{l}\text { Ignorancia } \\
\text { de las } \\
\text { restricciones } \\
\text { de las reglas }\end{array}$} & \multicolumn{2}{|c|}{$\begin{array}{l}\text { Aplicación } \\
\text { incompleta } \\
\text { de las } \\
\text { reglas }\end{array}$} \\
\hline & 32 & $91 \%$ & 69 & $84 \%$ & 2 & $67 \%$ \\
\hline $\begin{array}{l}\text { Past } \\
\text { continuous }\end{array}$ & 0 & $0 \%$ & 0 & $0 \%$ & 1 & $33 \%$ \\
\hline Past perfect & 3 & $9 \%$ & 13 & $16 \%$ & 0 & $0 \%$ \\
\hline $\begin{array}{l}\text { Past perfect } \\
\text { continuous }\end{array}$ & 0 & $0 \%$ & 0 & $0 \%$ & 0 & $0 \%$ \\
\hline
\end{tabular}

Tabla 4 Descripción general de errores por categoría

En sintonía con el análisis y su incidencia en las oraciones en 1) past simple, se encontró que la presencia del error intralingual dentro de esta categoría es a razón de diversos factores. En primera los errores de hipergeneralización, muestran que el estudiante generaliza la construcción de una oración en pasado cuando, por identificación, no sigue una misma fórmula para su construcción.

Se muestra que el estudiante, a pesar de hacer uso del auxiliar did, al mismo tiempo utiliza forma en pasado del verbo principal de la oración: but that didn't stopped us, what did she had that I didn't?, we didn't paid much attention. Lo anterior aborda la generalización de reglas al asumir que en todos los casos el verbo principal tiene que ser en pasado.

Por otro lado, se encontraron errores al asumir que la forma en pasado de cualquier verbo es a través de la terminación -ed; por ejemplo en: hurted, fighted, felted, leaved, knowed. No obstante, se deja ver el hecho que los estudiantes son capaces de identificar el verbo y en qué oraciones aplicarlo, es decir, ya cuenta con una noción generalizada. En segundo lugar, los errores que inciden en ignorancia de las restricciones de las reglas, se encontró que el estudiante insiste en mostrar una regla que no aplicaría para el uso del tiempo past simple. Es decir, ignora los momentos en los cuales debe dar uso al tiempo verbal.

Una forma de representar este tipo de error es utilizar el presente simple cuando dentro de la narrativa la coherencia reside en el uso del tiempo verbal pasado (past simple): I can rest my mind for a while, he get himself a new girlfriend, I meet some friends in university. En estas oraciones, se hace presente que el estudiante considera correcta la aplicación de los verbos can, get y meet como acciones llevadas a cabo dentro de la narrativa. Las oraciones correctas, de acuerdo con aquellas mencionadas previamente, deberían quedar de la siguiente forma: I could rest my mind for a while, he got himself a new girlfriend, I met some friends in university.

El otro ejemplo que conserva esta categoría, es debido a que el estudiante considera la forma del pasado past simple como figura conveniente. No obstante, no sería el recursos lingüístico apropiado: since I didn't have a vacation in a quite long time. Como se aprecia, el estudiante omite que hacia la 
forma y función para la composición del pasado existen restricciones. Es decir, realmente no se hablaría de la utilización del past simple, sino de una oración en past perfect: since I hadn't been on vacation in a quite long time.

Ocurre un fenómeno similar en la siguiente oración, pero en este caso el estudiante identifica el uso del auxiliar will. Al igual que en el ejemplo anterior, no es el tiempo verbal conveniente: I didn't know that will change my world. Como se aprecia en el ejemplo, no hace uso de la forma para la composición del segundo condicional, e ignora que existen restricciones que este tiempo conlleva. Es decir, el alumno no debería usar el verbo auxiliar will, sino del verbo auxiliar would para poder formar la oración en segundo condicional: I didn't know that would change my world.

Otra manera de sustentar esta falta de restricciones de las reglas, surge en dar uso de otros recursos que no van acorde con las intenciones de la oración. Esto expresa que el estudiante debe tener claro cuándo puede dar uso a ciertas formas condicionales dentro de una oración y cómo esta estructura enmarca un razonamiento lógico dentro de una oración en pasado: I didn't know that will change my world, his soul needed freedom or it will slowly die.

Dentro de los hallazgos se identificaron otras figuras de la omisión de ciertas reglas consideradas como parte de esta tipología. Se identificó el uso incorrecto del pasado del verbo to be: the look on cookies's eyes were like the one all kids wake up with. En este punto reconocer que el uso de esta regla obedece al factor numérico del sujeto en la oración, es decir, singular o plural. Dicho de otro modo, en la oración señalada el alumno considera como sujeto al sustantivo eyes, cuando en realidad el sujeto es look. La oración debe ser expresada de la siguiente manera: the look in Cookies' eyes, was like the one all kids wake up with. Si bien, el alumno conoce que existen las formas was y were, pero omite en qué contextos cada una de las dos formas deben ser aplicadas. Algo similar ocurre en: the world were to big for me. En esta oración, se ignoran ciertas reglas medulares: was/were.

Finalmente, se muestran errores de aplicación incompleta de las reglas para esta distinción es necesario indicar que el estudiante omite algún elemento particular dentro de la oración para su construcción en pasado. Dentro de los ejemplos: my mother got an incredible idea. En esta oración, algo que se reconoce para este tipo de errores, es la falsa selección del verbo puesto que por regla la oración pierde cierto sentido. La propuesta sugeriría que el verbo a utilizar es have (had) que reflejaría una función lógica en la oración.

En un segundo nivel, la representación del tiempo 2) past continuous comprende un solo error. El cuál a su vez, subyace dentro de la tipología de aplicación incompleta de las reglas. El ejemplo por discutir omite el uso de un verbo: we were going back to our hometown. Se muestra que el estudiante omite el uso del verbo to go en la oración, lo que se intenta expresar es una acción futura pero desde la perspectiva de una acción ocurrida en tiempo pasado, es decir: we were going to go back to our hometown.

En una tercera línea, el error para la conformación del 3) past perfect. La propuesta de identificación del error en lo que respecta a esta categoría verbal past perfect sugirió un índice de frecuencia inferior. Al igual que se menciona en la sección previa, el punto de partida refiere a los errores de hipergeneralización. Dentro de los datos recuperados en esta sección se encontró el siguiente ejemplo: And even though I had saw him a few weeks ago.

La construcción verbal anterior, deja ver con claridad la generalización de reglas hacia la construcción del past perfect. Se identificó que la versión correcta de la oración sería: and even though I had seen him a few weeks ago. Se comprende que el estudiante conoce el pasado del verbo see (saw) sin embargo, no conoce la forma en participio y consecuentemente tiende a relacionar el uso de la forma en pasado con la forma en participio.

En sintonía, dentro de las apariciones en el texto y la conformación del past perfect, se encontraron evidencias un tanto particulares. Mediante la oración: I have learn to step into pieces of my memory. Se comprende que la versión correcta de la oración sería: I have learned to step on pieces of my memory. En el caso particular del verbo learn, el estudiante asume que debería ser usado en presente, o bien en su forma base, sin importar que se haga uso del verbo have a modo de auxiliar, con el objetivo de formar el presente perfecto (no past perfect). En este punto, cabe aclarar que aun cuando este estudio no se centra en tiempos verbales del presente, para este ejemplo en específico el estudiante debe conocer la forma en pasado del verbo principal. En una tercera evidencia para esta tipología, se encontró el siguiente ejemplo: Have you ever use drugs? en la construcción realizada por el alumno se identificó que el posicionamiento correcto debería ser: Have you ever used drugs? En este sentido, la utilización para esta oración guarda estrecha relación con la explicación de la oración anterior. En donde a pesar de que el estudiante hace uso del verbo have a manera de auxiliar, el verbo principal de la oración (use) es usado en la forma base.

También se encontraron errores que corresponden a la ignorancia de las restricciones de las reglas, donde el estudiante insiste en mostrar una regla que no aplicaría para el uso del tiempo past perfect. Los estudiantes aplican oraciones con estructuras que no corresponden con el tiempo verbal requerido; por mostrar algunos ejemplos:

I had lived happy moments

I had learned how to treat people

I had tasted a lot of different food

Si bien es cierto, en las primeras tres oraciones el estudiante 
hizo uso de estructuras correspondientes con el tiempo verbal en pasado perfecto, dado el contexto, dichas oraciones deben ser usadas en presente perfecto. En la narrativa, el estudiante cuenta la forma en la que ha desempeñado labores en un restaurante y es una acción que ha llevado a cabo hasta el momento de realizar la prueba escrita. Por todo lo anterior y a pesar de que las oraciones están estructuradas correctamente, la aplicación a las situaciones contextuales descritas no es adecuada. Finalmente, no fueron encontrados errores de aplicación incompleta de las reglas para el past perfect orientados al aspecto intralingual.

\section{Análisis de la encuesta}

La encuesta tenía como objetivo principal la comprensión del uso e importancia de los recursos tecnológicos aplicados al aprendizaje de la lengua meta en el contexto. Dentro de los datos recopilados $\mathrm{y}$, que se obtuvieron de las 46 encuestas aplicadas, se destacan los siguientes elementos en el orden de los reactivos considerados.

El primer elemento en la encuesta refiere, 1) For me, the most difficult part in learning english language is, precisa de la identificación de los elementos más complejos para el estudiante en el desarrollo de la lengua:

\begin{tabular}{|c|c|c|c|c|c|c|}
\hline hem & Sonaties & Arondioy & Ustanây & Whinint & Grammer & Oeke \\
\hline For me, the nout diflewis & 245 & $11 \%$ & 6 & $\pi$ & 4856 & $4 \mathrm{~N}$ \\
\hline $\begin{array}{l}\text { part in learnivg Einelish } \\
\text { fanguere is }\end{array}$ & II & 5 & 3 & 3 & 22 & 2 \\
\hline
\end{tabular}

\section{Fígura 1. Ítem número 1 de la encuesta}

Tras identificar los resultados, la mayoría de los estudiantes responden que uno de los retos hacia el aprendizaje del inglés es el compuesto gramatical. En segundo momento, se parte de un cuestionamiento de percepción para el aprendizaje del inglés que, desde luego, valdría ponerse sobre la mesa tras posicionar a la lengua desde el factor motivacional.

\begin{tabular}{|c|c|c|c|c|c|}
\hline Them & $\begin{array}{l}\text { Shoungly } \\
\text { disogere }\end{array}$ & Desogres & Undecided & Agree & $\begin{array}{l}\text { Sorveb } \\
\text { eqree }\end{array}$ \\
\hline \multirow{2}{*}{ Inte Eighish } & $n_{6}$ & $2 \%$ & $31 \%$ & $41 \%$ & $20 \%$ \\
\hline & 0 & 1 & 14 & 19 & 12 \\
\hline
\end{tabular}

Fígura 2. Ítem número 2 de la encuesta

Se hace evidente un interés por la lengua pero, en el caso de los datos menores, sería interesante conocer por qué el estudiante no tiene un interés por la lengua. Para la aproximación a la gramática de la lengua, el cuestionamiento 3) I like English grammar, aborda si realmente existe no solo un interés por la lengua sino por las reglas y formas que deberá conocer el estudiante.

\begin{tabular}{|c|c|c|c|c|c|}
\hline Ttem & $\begin{array}{l}\text { Srewert } \\
\text { ifrugeres }\end{array}$ & Disagnee & Undecided & Agyee & $\begin{array}{l}\text { Sreventy } \\
\text { agrea }\end{array}$ \\
\hline \multirow[t]{2}{*}{ thes Foplist gravenar } & DA & $2 \%$ & $31 \%$ & $41 \%$ & 2656 \\
\hline & 0 & 1 & 14 & 19 & 12 \\
\hline
\end{tabular}

Fígura 3. Ítem número 3 de la encuesta

Dentro de los datos recuperados y, que con claridad se obtendrían elementos similares hacia el cuestionamiento anterior, se puede encontrar que mayormente sí existe un interés por el compuesto gramatical de la lengua y sus distintas representaciones. Sin embargo, para hacer un contraste puntual y para evidenciar las razones por las cuales no existe un interés por la lengua pero, tampoco por la gramática, se parte del cuestionamiento 4) I don't like English grammar because. En este ítem, de opción múltiple, se hace ver lo siguiente:

\begin{tabular}{|l|l|l|}
\hline Categorización del item & $\mathrm{F}$ & $\mathrm{P}$ \\
\hline Idon't feel engoged by the material used. & 13 & $28 \%$ \\
\hline Idon't like grammar lexsons, & 4 & $9 \%$ \\
\hline Idon't like the way my teacher executes the lesson. & 9 & $20 \%$ \\
\hline Idon't like doing grammar as a homework. & 8 & $17 \%$ \\
\hline Idon't see the practicality of studying grammar & 2 & $4 \%$ \\
\hline Other & 10 & $22 \%$ \\
\hline
\end{tabular}

Fígura 4. Ítem número 4 de la encuesta

Para el aprendizaje del compuesto gramatical, se encuentra la necesidad de una aproximación distinta a los recursos educativos como acceso y mediadores a este conocimiento. En las otras respuestas, se habla de la complejidad que tiene su comprensión, y la confusión existente al aprender este contenido. A continuación, se retoma la autoevaluación tras proponer 5) I consider my English language grammar to be.

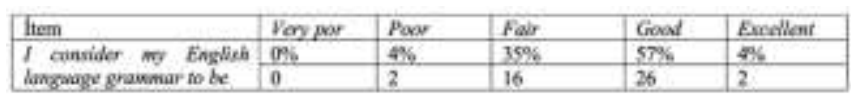

\section{Fígura 5. Ítem número 5 de la encuesta}

El hablar de un proceso de autoevaluación pone a discusión que los estudiantes tienen cierta conciencia de sus competencias y habilidades en la lengua; particularmente hacia el desarrollo de la gramática en la lengua extranjera. Por ello y en continuidad con este proceso de autoevaluación, el cuestionamiento 6) I know my strengths in English language presenta los aspectos siguientes:

\begin{tabular}{|c|c|c|c|c|c|}
\hline leem & $\begin{array}{l}\text { Srronght } \\
\text { divugrex }\end{array}$ & Disagnee & Undectiled & Agyer & $\begin{array}{l}\text { Sirrenght } \\
\text { Aure }\end{array}$ \\
\hline \multirow{2}{*}{$\begin{array}{l}1 \text { know my sirengths in } \\
\text { Fe fish language }\end{array}$} & $0 \%$ & $4 \%$ & $22 \%$ & $3 \% 6$ & $37 \%$ \\
\hline & 0 & 2 & 10 & 17 & 17 \\
\hline
\end{tabular}

\section{Fígura 6. Ítem número 6 de la encuesta}

Un punto de valor dentro del proceso de aprendizaje es reconocer, también, que el estudiante sea capaz de identificar cuáles son sus fortalezas para de ahí determinar sus debilidades. En cuanto al punto número 7) I know my weaknesses in English language los resultados arrojan:

\begin{tabular}{|c|c|c|c|c|c|}
\hline hem & $\begin{array}{l}\text { Srawkb } \\
\text { dingegere }\end{array}$ & Dicagrer & Linberided & Agre & $\begin{array}{l}\text { Simongb } \\
\text { Agree }\end{array}$ \\
\hline \multirow{2}{*}{ 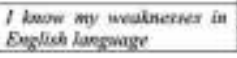 } & $\infty$ & $4 \mathrm{~N}$ & $13 \%$ & $48 \%$ & $35 \%$ \\
\hline & 0 & 2 & 6 & 22 & 16 \\
\hline
\end{tabular}

Fígura 7. Ítem número 7 de la encuesta

Dejar claro que el estudiante es un individuo reflexivo capaz de identificar sus fortalezas y debilidades; elemento que permitirá comprender en autonomía qué elementos deberá 
trabajar hacia el desarrollo de la lengua. En continuidad con la interpretación de los datos, uno de los aspectos para la comprensión del aspecto gramatical es la realimentación. Por ello, se considera el cuestionamiento: 8) I get good information from my teacher about how I am doing in class (if apply).

\begin{tabular}{|c|c|c|c|c|c|}
\hline item & $\begin{array}{l}\text { Sinongty } \\
\text { Aningree }\end{array}$ & Dinagrest & Thibribed & Agroe & $\begin{array}{l}\text { Sinoungty } \\
\text { Agree }\end{array}$ \\
\hline Iget good ingormation frow & $\infty$ & $11 \%$ & $25 \%$ & $41 \%$ & $20 \%$ \\
\hline $\begin{array}{l}\text { my nacher about how / aw } \\
\text { doing in clase of oppily }\end{array}$ & 0 & 5 & 13 & 19 & 9 \\
\hline
\end{tabular}

Fígura 8. Ítem número 8 de la encuesta

En lo que al ítem número 9 respecta, (I generally use correct grammar when I speak) se identifican los siguientes resultados. Sin embargo, antes de expresar los datos se debe considerar que esta discusión hacia el uso correcto de la gramática para este y el cuestionamiento siguiente se busca que el estudiante parte de una reflexión hacia el desarrollo de la lengua; tanto al aspecto oral como al escrito.

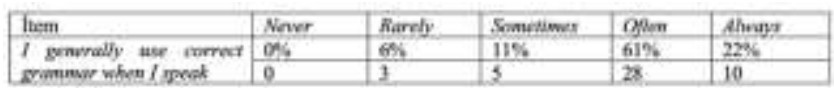

Fígura 9. Ítem número 9 de la encuesta

Siguiendo la línea del caso anterior, en la pregunta 10) I generally use correct grammar when doing written work, los estudiantes, en su mayoría, respondieron de manera afirmativa, en algunos casos respondieron "a menudo" y en otros casos "siempre". Con esto se infiere que, en lo que a la escritura concierne, los estudiantes tienen un poco más de tiempo para pensar acerca de las estructuras de las cuales harán uso, facilitando con esto, el uso correcto de cada tiempo verbal.

\begin{tabular}{|c|c|c|c|c|c|}
\hline \multirow{3}{*}{ 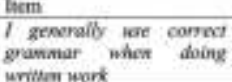 } & Newr & $R_{\text {axdy }}$ & Sanertimes & Coner & Athager \\
\hline & $\sigma_{6}$ & 68 & 148 & 515 & $2 n:$ \\
\hline & 0 & 3 & 7 & 25 & 14 \\
\hline
\end{tabular}

Fígura 10. Ítem número 10 de la encuesta

El reactivo número 11) Aspects in English Language grammar I consider I need to improve (select all that apply), la mayor área de oportunidad que presentan es en el uso de los gerundios, seguidos de las frases verbales, condicionales y superlativos.

\begin{tabular}{|c|c|c|c|c|c|}
\hline fiem & Propasinims & Gmands & Pasrsimule & Siperianive & Conifilionais \\
\hline \multirow{3}{*}{$\begin{array}{l}\text { Aipves is English } \\
\text { Lavgruge grawnar I } \\
\text { cumribler I nowd to } \\
\text { improve }\end{array}$} & $12(106)$ & $20(16)$ & $40 \%$ & $16(135)$ & 161 \\
\hline & $\begin{array}{l}\text { Plavasul } \\
\text { wofus }\end{array}$ & Adoectiver & Comparatives & $\begin{array}{l}\text { Modal } \\
\text { worly }\end{array}$ & colect \\
\hline & $19(15 \%)$ & 400 & 4080 & $16(13 \%)$ & 144 \\
\hline
\end{tabular}

\section{Fígura 11. Ítem número 11 de la encuesta}

Lo que interesaba demostrar para este espacio es comprender los elementos que busca mejorar. Es curioso ver que, desde la percepción del estudiante, que no se considera desarrollar mayormente los tiempos verbales; siendo que dentro de los datos obtenidos del primer instrumento se identificaron errores existentes en esta categoría.

En la identificación de cómo el estudiante tiene acceso, desde la representación del autoaprendizaje, a los distintos medios para el aprendizaje de la gramática: 12) I learned and practice English gramar. Los alumnos dejaron varias opciones al plasmar las formas en las que llevan a cabo el proceso de aprendizaje, sin embargo, también la forman en que llevan a cabo la práctica de la lengua.

\begin{tabular}{|c|c|c|c|c|c|c|}
\hline hem & $\begin{array}{l}\text { Ching } \\
\text { movial } \\
\text { molia }\end{array}$ & $\begin{array}{l}\text { Csing mabile } \\
\text { कrolications }\end{array}$ & $\begin{array}{l}\text { Warching } \\
\text { sutorialir }\end{array}$ & $\begin{array}{l}\text { Vuitiveg. } \\
\text { wnbuster. }\end{array}$ & $\begin{array}{l}\text { Taking } \\
\text { claner }\end{array}$ & $\begin{array}{l}\text { Cising } \\
\text { gramour } \\
\text { Bnote }\end{array}$ \\
\hline $\begin{array}{l}\text { lhearned Enpish } \\
\text { grawedr. }\end{array}$ & $\begin{array}{l}25 \\
(2250)\end{array}$ & 120196 & $4(439)$ & $14(13 \%)$ & $39(35 \%)$ & 17 (15\%) \\
\hline \\
\hline him & $\begin{array}{l}\text { Finking } \\
\text { wiek } \\
\text { Friends }\end{array}$ & $\begin{array}{l}\text { Using mobule } \\
\text { applikationt }\end{array}$ & $\begin{array}{l}\text { Watching } \\
\text { antorials }\end{array}$ & $\begin{array}{l}\text { Whatiching } \\
\text { udeos }\end{array}$ & $\begin{array}{l}\text { Taking } \\
\text { classes }\end{array}$ & $\begin{array}{l}\text { Doing } \\
\text { comises }\end{array}$ \\
\hline $\begin{array}{l}\text { I practice Enghish } \\
\text { gramewar }\end{array}$ & $\begin{array}{l}29 \\
21 \%)\end{array}$ & $18(135)$ & $3(2 \%)$ & $30(21 \%)$ & $34(2 \pi 6)$ & $\begin{array}{l}26 \\
\text { (1995) }\end{array}$ \\
\hline
\end{tabular}

Fígura 12. Ítem número 12 de la encuesta

Se observa que el aprendizaje mayormente parte de un ambiente presencial, así como la práctica de la lengua se hace en este ambiente formal. También se puede entender que este proceso parte de una socialización verbal con amigos pero, también a partir de distintos recursos tecnológicos que son parte de la vida cotidiana del individuo.

En lo que corresponde a 13) I ask for help to complete a determined English language task, más de la mitad de los encuestados respondieron que a veces, y el restante no refiere a pedir apoyo hacia la comprensión de algún tema:

\begin{tabular}{|c|c|c|c|c|c|}
\hline flem & Nener & Raredy & Sonvilimes & gom & Always \\
\hline I ark far hwlp so complese a & $6 \%$ & $37 \%$ & $46 \%$ & $4 \%$ & 796 \\
\hline $\begin{array}{l}\text { deterwined English } \\
\text { Bencwese tork }\end{array}$ & 3 & 17 & 21 & 2 & 3 \\
\hline
\end{tabular}

Fígura 13. Ítem número 13 de la encuesta

En el reactivo 14) I like learning English using technology, casi todos los estudiantes respondieron de manera afirmativa que les gusta aprender el idioma a través del uso de la tecnología:

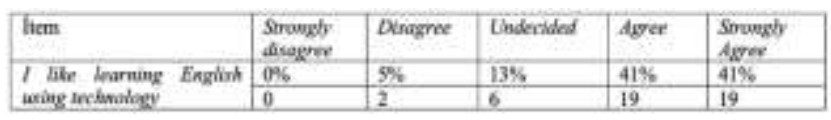

Fígura 14. Ítem número 14 de la encuesta

Este dato presenta el punto principal de esta investigación que radica, precisamente, en el uso de la tecnología para mejorar las habilidades gramaticales en la lengua. En el cuestionamiento 15) Devices I use to learn or practice English language, los alumnos se decantaron más por el uso del teléfono celular, y posteriormente por la computadora:

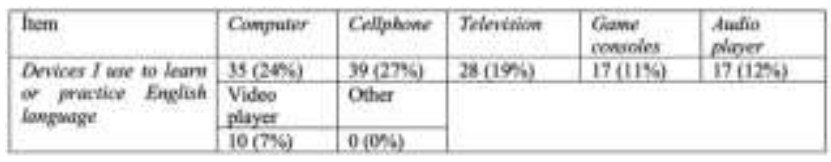


Fígura 15. Ítem número 15 de la encuesta

Se puede concluir que los estudiantes contestaron estas dos opciones al ser con las que comúnmente todos tienen contacto. Por otro lado, ligado al rubro anterior, el caso 16) According to the previous answer, how often I use this device, los alumnos comentan que hacen uso del recurso con cierta frecuencia:

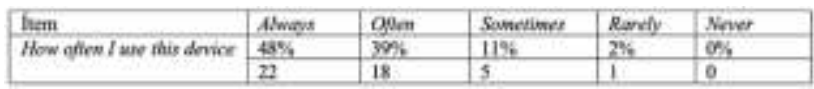

Fígura 16. Ítem número 16 de la encuesta

En el siguiente caso 17) The resources I like to use in English class (select all that apply), los datos encontrados fueron los siguientes:

\begin{tabular}{|c|c|c|c|c|c|}
\hline tem & Doous & Mories & Sange & Social mantig & Casformices \\
\hline \multirow{3}{*}{ 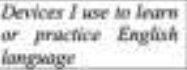 } & $2 \times(12 \%)$ & 330467 & $30(1 \%)$ & $28(12 \%)$ & $10(-4)$ \\
\hline & Photiacupers & Wideor & Aprticatians & Elect devicer & Hintricese \\
\hline & $22(96)$ & $31(135)$ & $15(68)$ & 16079 & $23(926)$ \\
\hline
\end{tabular}

Fígura 17. Ítem número 17 de la encuesta

Los resultados anteriores muestran que los estudiantes se inclinan, en su mayoría, por las películas, las canciones y los videos. Estos son los recursos que más les interesan al momento de llevar a cabo el proceso de aprendizaje.

Los cuestionamientos complementarios y para cerrar este apartado del recurso tecnológico y la encuesta, persigue destacar que, hacia la construcción de ambientes de aprendizaje, los accesos al recurso no se limitan al estar frente a una computadora de escritorio; por lo contrario habilitar espacios de consulta a través de cualquier dispositivo: computadora, tableta electrónica o celular. En el punto número 18) Mobile applications that I use to learn English grammar, se contemplaba la identificación de los espacios de acceso o de consulta por parte del estudiante.

\begin{tabular}{|c|c|c|c|c|c|}
\hline teem & $\begin{array}{l}\text { Rowema } \\
\text { Sane }\end{array}$ & Mind'snarks & Rabled & Mrasuliogent & D̂tent \\
\hline \multirow{2}{*}{ 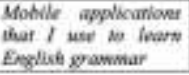 } & $6(1954)$ & $1(5 \%)$ & $0(0 \%)$ & $1(3 \%)$ & $1(5 \%)$ \\
\hline & $\frac{\text { Dundieges }}{\left.20<65^{5}\right)}$ & $\frac{\text { Momribe }}{2(7 \%)}$ & Ontwer & & \\
\hline
\end{tabular}

Fígura 18. Ítem número 18 de la encuesta

Los alumnos encuestados respondieron que las aplicaciones de las cuales hacen uso son: Rosetta Stone y Duolinguo. De esto, se puede inferir que los alumnos prefieren llevar a cabo el proceso de enseñanza a través de situaciones cotidianas y a través de la exploración de contenidos. Y en el último punto 19) Activities I would like to develop in English class (select all that apply):

\begin{tabular}{|c|c|c|c|c|c|}
\hline hem & $\begin{array}{l}\text { Grumenar } \\
\text { wrimen } \\
\text { crescles. }\end{array}$ & $\begin{array}{l}\text { Rowiling and } \\
\text { promunciation } \\
\text { exprites. }\end{array}$ & $\begin{array}{l}\text { Vocutwilary } \\
\text { esercines. }\end{array}$ & Connwations. & Presentunions. \\
\hline \multirow{3}{*}{$\begin{array}{l}\text { Actinitier I } \\
\text { would the to } \\
\text { denvlop in to } \\
\text { English clases }\end{array}$} & $20(15 \%)$ & $28(215)$ & $23(068)$ & $30(23 \%)$ & $15.112 \%)$ \\
\hline & $\begin{array}{l}\text { Actrvities } \\
\text { with } \\
\text { techowogical } \\
\text { nesources. }\end{array}$ & Other & & & \\
\hline & $15(11 \%)$ & $D(0 \times)$ & & & \\
\hline
\end{tabular}

\section{Fígura 19. Ítem número 19 de la encuesta}

Tras enunciar los distintos ítems, se entiende que los recursos tecnológicos propician un ambiente de aprendizaje idóneo para el estudiantado en formación. El estudiante es consciente de sus capacidades hacia el aprendizaje pero, también es reflexivo de las distintas áreas de oportunidad que tiene para el aprendizaje de la lengua meta y que desde luego desea trabajar por algún medio u otro.

\section{Producto}

El objetivo principal de esta sección es describir el producto final derivado de esta investigación. Se busca proveer a los estudiantes del tronco común de la Facultad de Idiomas de la Universidad Autónoma de Baja California, sede en Mexicali, con un recurso en línea mediante el cual se tenga acceso a explicaciones acerca de los tiempos verbales del pasado en inglés: past simple, past continuous, past perfect, past perfect continuous.

El contenido de la plataforma consiste en videos, explicaciones escritas y visuales, ejercicios de reafirmación (escritos y en línea), así como material adicional (actividades y material para el profesorado) con el que se podría llevar a cabo la práctica de los tiempos descritos. Los usuarios deberán acceder al siguiente enlace para poder ingresar a la página:

https://octavioquintero6.wixsite.com/languageteaching

Para simplificar la descripción de las partes que componen la página web, se destacarán las secciones más relevantes. En la página principal de la plataforma se encuentra un vídeo introductorio que explica en qué consiste la plataforma. Así mismo se muestran dos secciones principales: Every English Tense y Supplementary Material.

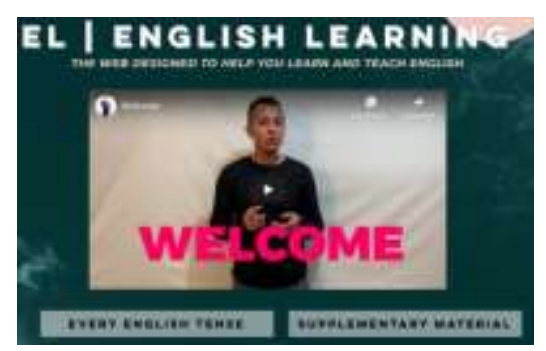

Fígura 20. Página de inicio plataforma

Por lo que refiere a la sección Every English Tense, básicamente su función es guiar al usuario hacia la comprensión de los tiempos verbales en pasado; acompañado de otros recursos. En la sección que no ampliaremos, Supplementary material, se podrán encontrar otros recursos como páginas web complementarias que refieren a ESL. 


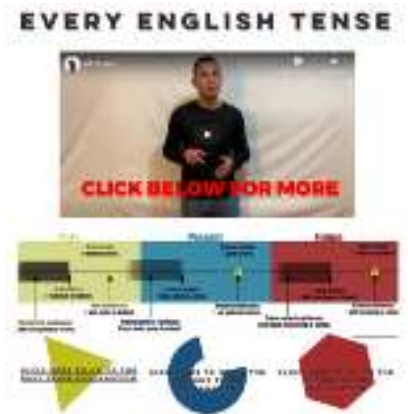

Fígura 21. Every English Tense

El punto principal, mismo que justifica el proceso realizado tanto de la prueba diagnóstica como la encuesta, es la sección past tenses. En esta sección se encuentra un vídeo con la explicación general de los tiempos verbales en inglés, donde posteriormente encontrarán explicaciones del tiempo verbal pasado: Simple, Continuous, Perfect y Perfect continuous.

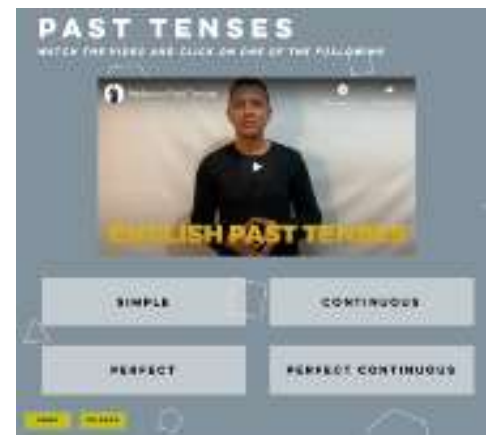

Fígura 22. Past tenses

Para resumir este apartado, las cuatro secciones comparten una estructura similar. En cada sección puede encontrar un vídeo con una descripción detallada del tiempo verbal pasado; acompañado de otros recursos complementarios: Written explanation, Visual explanation, Exercises, y la sección For teachers.
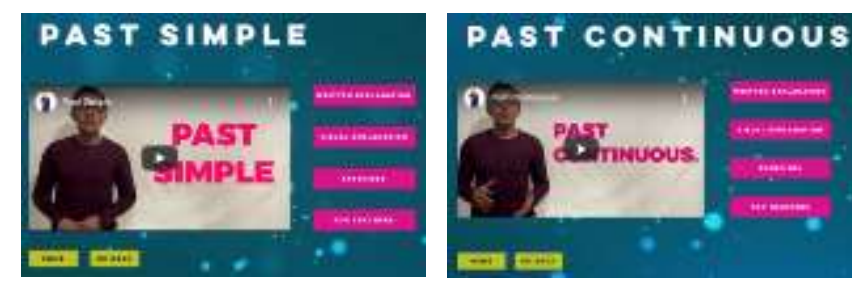

Fígura 23. Ejemplo sección específica del tiempo verbal

\section{Conclusiones}

En este texto se presentaron los principales datos que surgieron del análisis de errores y la encuesta. A partir de los instrumentos, se plantearon recursos tecnológicos para el desarrollo del tiempo verbal pasado en inglés. Tomando en cuenta distintos elementos como: vídeos, fichas descriptivas del tiempo verbal, elementos visuales, entre otros que se describieron anteriormente. El propósito fue desde luego dar respuesta a la pregunta de investigación inicial que refiere al uso correcto de estructuras gramaticales para la composición de la narrativa. Los datos obtenidos establecen que existen contenidos que deberán trabajarse hacia la consolidación de un nivel B1 de acuerdo a los parámetros del MCER.

Por su parte la tipología intralingual propuesta, siendo un acercamiento propio de esta investigación, constituye una falta de comprensión de la lengua. Donde el estudiante en formación debe entender a la lengua bajo ciertos criterios estructurados y que la lengua se rige por normas y reglas. Entendemos que en un aprendizaje aún en tránsito existen competencias y habilidades a desarrollar en la lengua meta, no obstante, es en este espacio temporal donde se requieren establecer los medios o recursos para favorecer el aprendizaje. En este sentido y dando respuesta a la segunda pregunta planteada, podemos partir que existe una diversidad de recursos apoyados en tecnologías que pueden implementarse en y fuera del aula.

Para construir un espacio basado en tecnologías, reiteramos, fue necesario establecer una base explicativa que parte de una necesidad formativa. Donde resulta dejar claro que cualquier contribución de esta índole parte de la contextualización, así como de la necesidad de proveer de recursos que favorezcan la consolidación de ciertos contenidos lingüísticos; para este caso un contenido gramatical. Sobre todo al reconocer que dentro de una etapa inicial de la formación profesional se requiere apoyar al estudiante mediante recursos pertinentes y auténticos.

Destacar finalmente que este tipo de investigaciones que dejan un producto sobre la mesa, cobran relevancia al insistir que no solo basta hacer una revisión del contexto e indicar las áreas de oportunidad, es proveer de nuevas oportunidades de aprendizaje que resultan facilitadoras a través del uso de las tecnologías. Estamos en el entendido que este espacio educativo elaborado para satisfacer la necesidad de la comprensión del tiempo verbal pasado es el inicio de futuras investigaciones y propuestas dentro de nuestro contexto formativo; sobre todo de reconocer la importancia que tiene la creación de distintos ambientes de aprendizaje.

\section{REFERENCIAS}

[1]Adell, J. (1997). Tendencias en educación en la sociedad de las tecnologías de la información. EDUTEC, Revista Electrónica de Tecnología Educativa(7). Obtenido de https://nti.uji.es/docs/nti/Jordi_Adell_EDUTEC.html

[2]Akbar, A. (2012). Error Analysis and Second Language Acquisition.Theory and Practice in Language Studies, 2(5), págs. 1027-1032. de 
https://pdfs.semanticscholar.org/2902/a09fba854bdbcfdeeb92a6591c9 9b5f918ab.pdf

[3] Bialystok, E. (1988). Levels of Bilingualism and Levels of Linguistic Awareness. Developmental Psychology, 24(4), 560-567. Obtenido de https://pdfs.semanticscholar.org/9957/e8524586a59c19b1c1f149106d8 444a489d3.pdf

[4] Bolitho, R., Carter, R., Hughes, R., Ivanic, R., Masuhara, H., y Tomlinson, B. (julio de 2003). Ten questions about language awareness. ELT Journal, 57(3), 251-259. Obtenido de http://sayqeeh.com/j/sade/4_language_awareness.pdf

[5]Beatty, K. (2010). Teaching and Researching: Computer-Assisted Language Learning. London: Pearson Education Limited. Obtenido de http://englishlangkan.com/produk/E\%20Book\%20Teaching\%20and\%2 0Researching\%20CALL\%20Ken\%20Beatty\%202011.pdf

[6]Canale, M. (1995). De la competencia comunicativa a la pedagogía comunicativa del lenguaje. Competencia comunicativa : documentos básicos en la enseñanza de lenguas extranjeras, 63-82. Obtenido de https://dialnet.unirioja.es/servlet/articulo? codigo $=574838$

[7] Chapelle, C., \& Hegelheimer, V. (2004). New perspectives on CALL for second language classrooms. The language teacher in the $21 \mathrm{st}$ century, 299-316.

[8] Corder, S. (1967). The Significance of Learner's Errors. Heidelberg: Julius Groos Verlag. Obtenido de https://files.eric.ed.gov/fulltext/ED019903.pdf

[9] Dulay, H., Burt, M., y Krashen, S. (1982). Language two. New York: Oxford University Press. Obtenido de https://www.academia.edu/7416848/_EBOOK_Language_Two_by_H eidi_Dulay_Marina_Burt_and_Staphen_Krashen_1982_source_BookF i.org

[10] Egbert, J., y Petrie, G. (2006). CALL Research Perspectives. New York: Routledge.

[11] Fasold, R., y Connor-Linton, J. (2013). An introduction to language and linguistics. Spain: Cambridge University Press. Obtenido de https://kupdf.net/download/an-introduction-to-language-andlinguistics_5af870f6e2b6f5d7353d7551_pdf

[12]Instituto Cervantes. (2002). Marco Común Europeo de Referencia para las Lenguas: Aprendizaje, Enseñanza, Evaluación. España: Ministerio de Educación, Cultura y Deporte, Subdirección General de Cooperación Internacional, para la edición impresa en español. Recuperado de http://cvc.cervantes.es/obref/marco

[13]González, D. y Ortíz, M. (2016). Storyboard como herramienta de apoyo didáctico en el desarrollo de competencias investigativas en estudiantes de educación superior. Caso: delimitación del problema de investigación. En A. Méndez, \& D. Gutiérrez, Estrategias de enseñanza y aprendizaje. Una mirada desde diferentes niveles educativos (pág. 144). Durango: Red Durango de Investigadores Educativos A. C. Obtenido de http://iunaes.mx/inicio/wpcontent/uploads/2017/02/Libro-9.pdf

[14] Hubbard, P. (2009). Computer Assisted Language Learning: Critical Concepts in Linguistics. New York \& London: Routledge.

[15]James, C. (2013). Errors in Language Learning and Use: Exploring Error Analysis. London: Routledge. Obtenido de https://bok.lat/book/3434470/9c9215?regionChanged

[16]Levy, M., y Stockwell, G. (2006). CALL dimensions: options and issues in computer assisted language learning. Language, learning and technology, 11(2), 27-30. Obtenido de https://www.researchgate.net/publication/237710614_CALL_dimensio ns_options_and_issues_in_computer_assisted_language_learning

[17] Lyster, S. (2001). Language and reading-Development and disorders. En S. Lyster, Special education needs education: An introduction. Unipub.

[18] Martín, E. (2012). Presente y futuro de la enseñanza de lenguas asistida por ordenador: ¿El final de una era? Revista de Lingüística y Lenguas Aplicadas, 7, 203-212. Obtenido de
https://www.researchgate.net/publication/259981090_Presente_y_futur o_de_la_ensenanza_de_lenguas_asistida_por_ordenador_El_final_de una_era

[19] Martín, E., Arjonilla, A., Atienza, E., Castro, M., Higueras, M., Camiruaga, M., y Vañó, A. (2005). Diccionario de términos clave de ELE del CVC. Universidad de Sevilla.

[20] Navarro, W. (2011). Las TIC en el sistema educativo colombiano. REVISTA VINCULOS, 8(1), págs. 150-156. Obtenido de https://revistas.udistrital.edu.co/index.php/vinculos/article/view/4175/5 835

[20]Richards, J. (1975). Error Analysis: Perspectives on Second Language Acquisition. London: Routledge. Obtenido de https://www.taylorfrancis.com/books/9781315836003

[21]Richards, J., y Reppen, R. (2014). Towards a Pedagogy of Grammar Instruction. RELC Journal, 45(1), 5-25. Obtenido de https://www.professorjackrichards.com/wp-content/uploads/Grammarinstruction.pdf

[22]Roehr, K. (2007). Metalinguistic Knowledge and Language Ability in University-Level L2 Learners. Applied Linguistics, 29(2), 173-199. Obtenido de https://core.ac.uk/download/pdf/4188071.pdf

[23]Roldán, E. (1979). La técnica del análisis de errores. Documentos Lingüísticos y Literarios, 3, págs. 47-52. Obtenido de http://www.humanidades.uach.cl/documentos_linguisticos/docannexe. php?id=182

[24] Rustipa, K. (2011). Contrastive Analysis, Error Analysis, Interlanguage and the Implication to Language Teaching. Ragam Jurnal Pengembangan Humaniora, 11(1). Obtenido de https://pdfs.semanticscholar.org/255e/bdf90f8353aac8347ec8fcbe7e0f 66fa92f2.pdf

[25]Sañudo, J. (2013). La enseñanza de la gramática y el uso del corrector gramatical (grammar checker). UNED, Facultad de Filología. Madrid: Facultad de Filología, UNED. Obtenido de https://eprints.ucm.es/27320/1/tfm.pd 